\title{
A Cognição Social e o Córtex Cerebral
}

\author{
Judith Butman ${ }^{1}$ \\ Ricardo F. Allegri ${ }^{2}$ \\ Universidade de Buenos Aires, Argentina
}

\begin{abstract}
Resumo
A cognição social é o processo que orienta condutas frente a outros indivíduos da mesma espécie. Várias estruturas cerebrais têm um papel chave para controlar as condutas sociais: o córtex pré-frontal ventromedial, a amígdala, o córtex somatosensorial direito e a ínsula. O córtex pré-frontal ventromedial está comprometido com o raciocínio social e com a tomada de decisões; a amígdala com o julgamento social de faces; o córtex somatosensorial direito, com a empatia e com a simulação; enquanto que a insula, com a resposta autonômica. Estes achados estão de acordo com a hipótese do marcador somático, um mecanismo específico por meio do qual adquirimos, representamos ou memorizamos os valores de nossas ações. Estas estruturas cerebrais atuam como mediadores entre as representações perceptuais dos estímulos sensoriais e a recuperação do conhecimento que o estímulo pode ativar. O sistema límbico é a zona limítrofe; nela, a psicologia se encontra com a neurologia. A correta sincronização destas zonas e estruturas, no adulto, é a chave para uma situação livre de patologia.

Palavras-chave: Cognição social; pré-frontal; amígdala; razão; emoção; córtex cerebral.
\end{abstract}

\section{Social Cognition and the Brain Cortex}

\begin{abstract}
Social cognition refers to the processes that subserve behavior in response to other individuals of the same species. Several brain structures play a key role in guiding social behaviors: ventromedial prefrontal cortex, amygdala, right somatosensory cortex and insula. The ventromedial prefrontal cortex is most directly involved in social reasoning and decision making; the amygdala in social judgment of faces, the right somatosensory cortex in empathy and simulation and the insula in autonomic responses. These findings are corresponding to the somatic marker hypothesis, particular mechanism by which we acquire, represent and retrieve the values of our actions. These brain structures appear to mediate between perceptual representation of social stimuli and retrieval of knowledge that such stimuli can trigger. The limbic system is the border zone where psychology meets neurology. The synchronization of these functions and structures is the key for a human being free of pathology.

Keywords: Social cognition; prefrontal; amygdala; reasoning; emotion; brain cortex.
\end{abstract}

A cognição social é o processo neurobiológico que permite tanto humanos como animais interpretar adequadamente os signos sociais e, conseqüentemente, responder de maneira apropriada. Outra definição poderia corresponder ao processo cognitivo que elabora a conduta adequada em resposta a outros indivíduos da mesma espécie, especificamente, aqueles processos cognitivos superiores que sustentam as condutas sociais extremamente diversas e flexíveis (Adolphs, 1999). As estruturas anatômicas implicadas nestes processos, baseando-se em estudos experimentais com animais e com pacientes com lesões cerebrais são: a amígdala, o

\footnotetext{
${ }^{1}$ Endereço para correspondência: Servicios de Neurología y de Neuropsicologia - Cemic - CONICET - Galvan 4102 (1431) Buenos Aires Argentina - Fax: (54-11) 4922-9833.E-mail: allegri@jede.net
}

córtex pre-frontal ventromedial, a ínsula e o córtex somatosensorial direito.

\section{Córtex Pré-Frontal Ventromedial}

A participação do córtex pré-frontal na cognição social é conhecida desde o caso de Phineas Gage que, logo após um acidente de trabalho numa ferrovia, tornou-se despreocupado e passou a ter uma conduta social inapropriada. Nas palavras de seu médico, Harlow: "Destruiu-se o equilibrio entre suas faculdades intelectuais e suas inclinações animais." (Damasio , 1994). Em 1888, Leonor Welt publica em seu doutorado uma correlação entre a lesão orbital vizinha à linha média e mudanças de caráter, com base na observação de 12 pacientes, sendo um deles Phineas Gage. Kleist, em 1931 (citado em Outes, 
Florian \& Tabasso, 1997), observou que os pacientes com lesões em áreas orbitárias mostravam uma dificuldade em inibir seus impulsos. Este autor relaciona o córtex orbital medial (área 11 de Brodmann) ao "eu social".

Vários autores (Goldar, 1993; Mega, Cummings, Salloway \& Mallory,1997; Tucker, Luu \& Pribram, 1995) referem que o neocórtex ventral e as estruturas paralímbicas ventrais ocupam-se de outorgar um valor aos sentimentos e aos atos que executa o neocórtex dorsal. Deve-se fazer um esclarecimento anatômico: estruturas paralímbicas ventrais correspondem ao córtex pré-frontal ventromedial, amígdala, ínsula ventral e pólo temporal (Mesulam, 2000). Já o córtex pré-frontal orbital, mais anterior e lateral, corresponde ao neocórtex (Nauta, 1971) e, segundo Cummings (1995), sua lesão também ocasiona um transtorno nas condutas sociais.

O estudo de pacientes lesionados revela que pacientes com lesão pré-frontal têm dificuldades na tomada de decisões e no raciocínio social. Segundo Damasio (1994), tomar decisões é escolher uma opção de resposta entre as muitas possíveis num determinado momento e em uma determinada situação. Supõe conhecer: (1) a situação que exige tal decisão; (2) as distintas opções de ação; e, (3) as conseqüências imediatas ou futuras de cada uma das ações. Realizar estes passos através de uma perspectiva lógica dedutiva levaria muito tempo. Por essa razão, Damasio propõe a hipótese do marcador somático: uma situação se une a um estado somático específico, e é este padrão somatosensorial, ou marcador somático que qualifica uma situação como boa ou má. O estado somático dirige a atenção para eventuais conseqüências negativas das condutas, tornando as decisões mais rápidas e efetivas. Uma lesão no córtex pré-frontal ventromedial ocasiona, nos pacientes, falhas na utilização de sinais somáticos ou emocionais para guiar a conduta. Assim, eles se mostram alheios às futuras conseqüências de seus atos e atuam segundo suas perspectivas imediatas. Em outras palavras, o fenômeno do marcador somático corresponde ao prevenir-se para seguir sendo o que implica poder sentir desde as entranhas o chamado interoceptivo de perigo (Goldar, 1979).

Numerosos investigadores têm provado esta hipótese usando o paradigma de jogo de cartas. Bechara, Damasio, Damasio e Anderson (1994) e Bechara, Damasio, Tranel e Damasio $(1996,1997)$ demonstraram que pacientes com estas lesões preferem as jogadas mais perigosas em suas apostas. Entretanto, neles não é desencadeada uma resposta eletrodérmica ante à expectativa de ganhar ou perder, o que ocorre com os controles. Isso sugere que não se encontra ativado o marcador somático adequado, ou seja, suas escolhas no jogo eram alheias às futuras conseqüências de seus atos.

Para destacar que o lobo pré-frontal não é uma unidade funcional, Bechara, Damasio, Tranel e Damasio (1998) também demonstraram uma dissociação dupla: os pacientes com lesão pré-frontal dorsolateral direita teriam déficits nas tarefas que envolviam a memória de trabalho, mas teriam uma resposta eletrodérmica antecipatória adequada ante à expectativa de ganhar ou perder, no paradigma do jogo de cartas. Entretanto, pacientes com lesões pré-frontais ventromediais bilaterais rendem bem nas tarefas que envolvem a memória de trabalho, mas têm abolido a resposta eletrodérmica antecipatória ante à expectativa de ganhar ou perder um jogo de cartas.

Seguindo esta linha de raciocínio, no que diz respeito à dissociação das distintas funções pré-frontais e sua correlação anatômica, Davidson (1999) propõe que, assim como no córtex pré-frontal dorsolateral encontra-se a representação cognitiva da meta de uma ação na ausência de seu desencadeante imediato (o que todos conhecemos como memória de trabalho), no córtex pré-frontal ventromedial, encontra-se a representação emocional da meta de uma ação, na ausência de seu desencadeante imediato. Essa representação pode ser denominada memória de trabalho emocional. Como o córtex pré-frontal ventromedial está interconectado com o córtex orbital lateral, e este com o pré-frontal dorsolateral, a memória de trabalho emocional interatua com a memória de trabalho convencionalmente conhecida por todos.

O modelo atencional de Norman e Shallice (1986), ao explicar as funções do córtex pré-frontal, propõe a existência de um Sistema Supervisor Atencional, consciente e explícito, encarregado das condutas não-rotineiras e um Programa de Contenção, automático e implícito, que intervém na manutenção das condutas sociais e emocionais apropriadas ao meio. Este programa de contenção envolve uma espécie de atenção dirigida para componentes emocionais. Se pensarmos na organização anatômica pré-frontal ventromedial, podemos deduzir que ela está intimamente ligada aos núcleos basais de Meynert e núcleos da Banda Diagonal de Broca, que regulam a focalização da atenção por suas eferências para o tálamo, não só através de projeções colinérgicas, mas também através de gabaérgicas e glutamatérgicas. Também se relaciona com as estruturas dorsolaterais executivas e áreas sensoriais, assim como, canaliza a atenção para componentes que regulam o estado afetivo, como a amígdala (Heimer, Olmos, Alheid \& Zaborszky, 1991; Zaborszky, Gaykema, Swanson \& Cullinan, 1997; 
Zaborszky, Pang, Somogyi, Nadasdy \& Kallo, 1999) e o córtex cingular subcaloso (Bush, Luu \& Posner, 2000).

Quanto ao raciocínio social, realizaram-se estudos com o paradigma de Wason, que avalia o raciocínio dedutivo. Tal paradigma consiste em dar ao paciente uma frase condicional, do tipo "Se P, então Q", em um contexto que pode ser abstrato (por exemplo, "se um aluno mostra uma nota de 10, então a carta deve ter o número 3") ou ter relação com situações sociais (por exemplo, "se você toma cerveja, então deve ser maior de 18 anos"). O paciente deve decidir se as frases são verdadeiras ou falsas, enquanto lhe é mostrada a situação em um desenho. Os pacientes com lesão pré-frontal ventromedial rendem pouco quando têm que decidir acerca de situações sociais. Mas se o córtex pré-frontal dorsolateral está ileso, rendem bem quando têm que decidir acerca de uma situação abstrata, mostrando assim uma dissociação (Adolphs, 1999).

\section{Amígdala}

Desde os experimentos de Kluver e Bucy (1939), em que a extirpação bilateral da amígdala nos macacos produziu mudanças emocionais (como hipersexualidade, cegueira psíquica, falta de reatividade ante estímulos naturalmente perigosos ou rechaço social por parte dos macacos sãos), a amígdala despertou o interesse por sua intervenção na cognição social. Ela intervém elaborando uma avaliação cognitiva do conteúdo emocional de estímulos perceptivos complexos. Segundo Emery e Amaral (2000), o núcleo basal, por ser o de maior interconexão com o córtex pré-frontal ventromedial, atua no pareamento de sinais sociais com o contexto social apropriado.

A percepção do estado emocional de uma face é uma das funções perceptivas complexas mais estudadas. Haxby e colaboradores (2000) propõem que a percepção dos aspectos que se alteram na face (os quais seriam os sinais mais importantes para uma correta interpretação dos signos sociais, como a expressão emocional do pânico) é processada no sulco temporal superior e na amígdala, sobretudo na direita. A amígdala, por suas eferências que saem do núcleo central atingindo o hipotálamo e o tronco cerebral, é capaz de desencadear a resposta hormonal e neurovegetativa de estresse. Por outro lado, devido à sua conexão com o núcleo basal de Meynert, consegue modular a direção da atenção para o estímulo perigoso (LeDoux, 2000). A amígdala recebe aferências sensoriais talâmicas e de áreas sensoriais de associação (Rojas, Outes \& Goldar, 1998). Por outro lado, manda eferências para áreas sensoriais primárias antes de ocorrer a representação cortical do estímulo. Deste modo, ela deve regular, de uma forma dirigida, o que o córtex sensorial processa. Além do que, também mediante esta via, modula a direção da atenção para o estímulo perigoso. A produção, na amígdala, do efeito de potenciação em longo prazo pode explicar sua participação nos processos de ansiedade e estresse pós-traumático, nos quais as associações entre sinais perigosos e a resposta de estresse são aprendidos e reforçados, ocasionando os sintomas somáticos de ansiedade.

O processo de direção do olhar tem um peso preponderante na cognição social, não apenas em humanos (Emery, 2000). Discute-se o predomínio do hemisfério direito no processamento emocional de expressões faciais (percepção e produção da expressão emocional). Isto porque, ao atribuir maior expressividade emocional ao hemirrosto esquerdo do que ao hemirrosto direito, com o paradigma de figuras quiméricas (Sackheim, Gur \& Saucy 1978), Ross (1998) propôs que existe uma maior capacidade de percepção do estado emocional através do processamento da metade superior da face (olhos, olhar) do que da metade inferior (boca), atribuindo méritos à máxima popular que diz: "a alma de um indivíduo se revela por seus olhos".

Tais achados vão de encontro com a teoria da mente, ou seja, a capacidade de atribuir uma mente a outro indivíduo; ter habilidade de entender o papel de outros indivíduos, assim como de entender outros pontos de vista ou atribuir uma intenção a outra pessoa. Essa capacidade é fundamental na cognição social (Voeller, 1998). Os pacientes com autismo, que possuiriam anormalidades estruturais ou funcionais na amígdala, não têm capacidade de atribuir um estado mental ou inferir uma emoção em outra pessoa através do olhar. Isso foi demonstrado através de estudos funcionais e deu lugar à teoria do transtorno amigdalino no autismo (BaronCohen e cols, 1994, 2000). Também foi observado esse transtorno em pacientes com lesão amigdalina (sobretudo para o reconhecimento da expressão emocional do medo) e em esquizofrenia (Adolphs e cols., 1994; Adolphs, Tranel, Damasio \& Damasio, 1992; Broks e cols., 1998)

\section{Córtex Somatosensorial e Ínsula}

A capacidade de empatia ou a habilidade de detectar o que outra pessoa sente é medida pela capacidade de poder reproduzir em nosso próprio organismo um estado emocional similar. Para isso, devem estar preservados os mecanismos de interpretação de signos emocionais relevantes, descritos nos capítulos anteriores, bem como o córtex somatosensorial direito e a ínsula (Caselli, 1997). Se quisermos investigar a capacidade de um indivíduo 
em interpretar a expressão emocional de uma face, uma das maneiras possíveis é reproduzir a expressão da face no próprio organismo (através do córtex somatosensorial direito e ínsula) e detectar o sentimento que desencadeia (Adolphs, 1999). Nessa situação, os pacientes com lesão nestas áreas apresentam transtornos no juízo emocional de faces (Blonder, Bowers \& Heilman, 1991; Ross, 1994).

Charles Darwin propôs que certas emoções têm uma base neural inata, já que são expressas de maneira universal através das distintas culturas. Elas corresponderiam às emoções primárias: nojo, medo, pânico, tristeza, surpresa, interesse, felicidade e desgosto. Todas essas emoções têm sua base neural nas estruturas citadas nesta revisão. À medida que a criança cresce, de maneira normal aprende a manipular estas emoções conforme as normas e expectativas sociais (Ross, 1998), desenvolvendo uma correta cognição social. Assim, o córtex pré-frontal ventromedial permite uma integração entre a percepção de uma emoção e a resposta que desencadeia, seja uma conduta complexa elaborada pelo neocórtex orbitário, seja uma resposta autonômica ou motora (incluída a atenção) através das eferências amigdalinas. Por outro lado, o córtex somatosensorial direito e a ínsula permite uma correta manipulação da informação necessária para a interpretação e expressão emocional da face e, sobretudo, do olhar (tarefa que realiza juntamente com a amígdala).

As lesões neurológicas (vasculares, tumorais, degenerativas ou traumáticas) do lobo frontal determinam um transtorno mais ou menos evidente da "conduta social", o qual foi mal detectado pela neurologia clássica. Muitas patologias psiquiátricas também poderiam ser interpretadas a partir desta definição (como por exemplo, a esquizofrenia paranóide, o autismo e até uma fobia ou um transtorno de ansiedade generalizada). Limbo quer dizer margem ou borda, e o sistema límbico, que é a base neural desta conduta, é a fronteira entre a neurologia e a psiquiatria (Mega, Cummings, Salloway \& Mallory 1997). A correta sincronização e a ausência de lesão destas áreas coloca o adulto numa situação livre de patologia, que se reflete na máxima de Marco Aurélio (ano 121-180 a.C.): "uma mente tranqüila significa não mais que uma mente bem ordenada"

\section{Referências}

Adolphs R., Tranel D., Damasio H. \& Damasio A. (1994). Impaired recognition of emotion in facial expressions following bilateral damage to the human amygdala. Nature, 372, 669-672.

Adolphs R. (1999). Social cognition and the human brain. Trends in Cognitive Sciences 3, 469-479.

Archer J., Hay D. C. \& Young A.W. (1992). Face processing in psychiatric condition. British Journal of Clinical Psychology, 31, 45-61.
Baron-Cohen S., Ring H. A., Moriarty J., Schmitz B., Costa D. \& Ell, P. (1994). Recognition of mental state terms: Clinical findings in children with autism and functional neuroimaging study of normal adults. British Journal of Psychiatry, 164, 640-649.

Baron-Cohen S., Ring H. A., Bullmore E. T., Wheelwright S., Ashwin C. \& Williams S. C. R. (2000). The amygdala theory of autism. Neurosciences and Biobehavioral Review, 24, 355-364.

Bechara A., Damasio A., Damasio H. \& Anderson S. W. (1994). Insensitivity to future consequences following damage to human prefrontal cortex. Cognition, 50, 7-12.

Bechara A., Damasio H., Tranel D. \& Anderson S.W. (1998). Dissociation of working memory from decision making within the human prefrontal cortex. Journal of Neuroscience, 18, 428-437.

Bechara A., Damasio H., Tranel D. \& Damasio A. (1996). Failure to respond autonomically to anticipated future outcomes following damage to prefrontal cortex. Cerebral Cortex, 6, 215-225.

Bechara A., Damasio H., Tranel D. \& Damasio A. (1997). Deciding advantageously before knowing the advantegeous strategy. Science, 275, 12931295.

Blonder L.X., Bowers D. \& Heilman K. M. (1991). The role of the right hemisphere in emotional communication. Brain, 115, 1114-1127.

Broks P., Young A. W., Maratos E. J., Coffey P. J., Callder A. J., Isaac C., Mayes A. R., Hodges J. R., Montaldi D., Cezayirli E., Roberts N. \& Hadley D. (1998). Face processing impairments after encephalitis: Amygdala damage and recognition of fear. Neuropsychologia, 36, 59-70.

Bush G., Luu P. \& Posner M. (2000). Cognitive and emotional influences in anterior cingulate cortex. Trends in Cognitive Sciences, 4, 215-222.

Caselli R. (1997). Tactile agnosia and disorders of tactile perception. Em T. E. Feinber \& M. J. Farah (Orgs.), Behavioral neurology and neuropsychology (pp 277-288). New York: McGraw-Hill.

Cumming J. L. (1995). Anatomic and behavioral aspects of frontal-subcortical circuits. Annals of the New York Academy of Science, 769, 1-13.

Damasio A. (1994). El error de Descartes. Santiago, Chile. Editorial Andrés Bello.

Davidson R. (1999). The functional neuroanatomy of emotion and affective style. Trends in Cognitive Sciences, 3, 11-21.

Emery N. J. (2000). The eyes have it: The neuroethology, function and evolution of social gaze. Neurosciences and Biobehavioral Revien, 24, 581604.

Emery N. \& Amaral D. (2000). The role of the amygdala in primate social cognition. Em R. Lane \& L. Nadel (Orgs.), Cognitive neurosciencie of emotion (pp. 570-600). Oxford: Oxford University Press.

Goldar J. C. (1979). Mecanismos cerebrales y esquizofrenia. Neuropsiquiatría (Arg.), 10, 3-23.

Goldar J. C. (1993). Anatomía de la mente. Buenos Aires: Ediciones Salerno.

Haxby J. V., Hoffman E. A. \& Gobbini M. I.(2000). The distributed human neural system for face perception. Trends in Cognitive Sciences, 4, 223233.

Heimer L., Olmos J., Alheid G. \& Zaborszky L. (1991). "Perestroika" in the basal forebrain: Opening the border between neurology and psychiatry. Program in Brain Research, 87, 109-165.

Kluver H. \& Bucy P. C. (1939). Preliminary analysis of functions of the temporal lobes in monkeys. Archives of Neurology and Psychiatry, 42, 979-1000.

LeDoux J. (2000). Cognitive-emotional interactions: Listen to the brain. Em R. Lane \& L. Nadel (Orgs.), Cognitive neurosciencie of emotion (pp.129155). Oxford: Oxford University Press.

Mega M., Cummings J.L., Salloway S. \& Mallory P. (1997). The limbic system: An anatomic, phylogenetic, and clinical perspective. Journal of Neuropsycbiatry and Clinical Neurosciences, 9, 315-330.

Mesulam M. (2000). Principles of behavioral and cognitive neurology. Oxford: Oxford University Press.

Nauta W. (1971). The problem of the frontal lobe: A reinterpretation. Journal of Psycbiatry Research, 8, 167-187. 
Norman, D. A. \& Shallice, T. (1986). Attention to action: Willed and automatic control of behavior. Em R. Davidson, G. Schwartz \& D. Shapiro (Orgs.), Consciousness and self-regulation: Advances in research and theory (pp. 1-18). New York: Plenum.

Outes D. L., Florian L. \& Tabasso J. V. (1997). Kleist K. Diez. Comunicaciones. Introducción a las Localizaciones Cerebrales en Neuropsiquiatria. Buenos Aires: Editorial Polemos.

Rojas D. R., Outes M. \& Goldar J. C. (1998). Sobre algunos fascículos del cerebro mediobasal. Alcmeón, 7, 131-165.

Ross, E. D., Horman, R. W. \& Buck, R. (1994). Differential hemispheric lateralizaion of primay and social emotions: Implications for developing a comprehensive neurology for emotion, repression, and the subconscious. Neuropsychiatry, Neuropsychology and Behavioral Neurology, 7, 1-19.

Ross, E. D. (1998, Abril/Maio). Hemispheric control of facial expression and deceitful behaviours. American Academy of Neurology, 50th Annual Meeting (pp. 33-52). Minneapolis, Minnesotta: USA.
Sackheim, H. A., Gur, R. C. \& Saucy, M. C. (1978). Emotions are expressed more intensely on the left side of the face. Science, 202, 434-436.

Tucker, D. M., Luu P. \& Pribram K. H. (1995). Social and emotional selfregulation. Em Annals of the New York Academy of Science, 769, pp.213240

Voeller, K. J. (1998, Abril/Maio). Developmental disorders of facial processing. American Academy of Neurology, 50th Annual Meeting (pp. 63-97). Minneapolis, Minnesotta, USA.

Zaborszky L., Gaykema, R. P., Swanson D. J. \& Cullinan, E. (1997). Cortical input to the basal forebrain. Neuroscience, 79, 1051-1078.

Zaborszky, L., Pang, J., Somogyi, J., Nadasdy, Z. \& Kallo, I. (1999). The basal forebrain corticopetal system revisited. Annals of the New York. Academy of Science, 877, 339-367.

Recebido: 28/03/2001

Revisado: 24/05/2001

Aceite final: 12/06/2001

Sobre os autores:

Judith Butmann é Médica Psiquiatra, Residente de Investigação Médica, Direção de Capacitação, GCBA. Psiquiatra do Laboratório de Memória Hospital Zubizarreta; Docente do Curso de Atualização em Neuropsicologia da Faculdade de Medicina, Universidade de Buenos Aires.

Ricardo F. Allegri é Médico Neurologista. Investigador Clínico do CONICET (Conselho Nacional de Investigações Científicas e Tecnológicas), Chefe do Serviço de Neuropsicología do CEMIC Hospital associado à Universidade de Buenos Aires. Docente Autorizado - Neurologia - Universidade de Buenos Aires. Diretor da Revista Neurológica Argentina. 